\title{
NUMERICAL STUDY ON HOMOGENOUS CONDENSATION OF STEAM IN CONVERGING DIVERGING NOZZLE
}

\author{
Sanju Hosur H', Anjaneyulu², K V Muralidharan ${ }^{3}$ \\ ${ }^{I} I V^{\text {th }}$ semester, MTech, Thermal Power Engineering, MVJCE Bengaluru \\ ${ }^{2}$ Manager-CFD Development, Fluidyn Consultancy Pvt Ltd Bengaluru \\ ${ }^{3}$ Department of Mechanical Engineering, MVJCE Bengaluru
}

\begin{abstract}
Steam is used as working fluid in power generation industry. Due to high pressure variations the steam condenses and forms two phase mixture. The condensation process in a turbo machine is in reality an essential random and unsteady phenomenon. The wet steam causes physical damage to the turbine blades and reduces its performance. It is very important for a turbine or nozzle designer to understand the behavior of this multiphase flow. Experimental prediction of condensation and variations in the steam flow parameters is difficult and costly. Hence in this work a comparison is made between equilibrium and non equilibrium mathematical models to predict properties of steam in converging diverging nozzles using a CFD tool FLUIDYN MP. The results are compared with the values in from literature work. Since the high speed involvement the steam stays in supercooled region and condensation initiates when the pressure drops below the Wilson line. Results of non equilibrium models were better and closer to experimental values when compared to equilibrium model.
\end{abstract}

\section{INTRODUCTION}

Understanding of wet steam flows in power plant turbines is a major concern for turbine designers and electricity producers. They have to solve important issues like controlling performances and machine life, evaluating new designs or the risks due to the enlargement of operating ranges, predicting and optimizing the maintenance costs. However it is difficult to produce a perfect design, as many different physical phenomena has to be considered while designing. The turbines operate at high pressure, the steam may be diphasic but the condensation appears in low pressure stages and accurate prediction of nucleation and growth of the droplet is difficult. Earlier the turbine designers did not considered the wetness into account, they thought that both phases are in thermal equilibrium this may lead to inaccuracies in loses. From rough estimation of wetness loses, it is found that one percent wetness increases loses by one percent [1]. The droplets those formed during the condensation, cause erosion and corrosion of turbine blades by their centrifugal impact and corrosive nature.

The flow inside the turbine is highly unsteady, many works were carried to solve issues related to unsteadiness, turbulence prediction and wetness prediction is less regarded. Thermodynamic equilibrium models are sufficient to deal with condensation, but one has to consider the non equilibrium models [2] because of delay in condensation. Steam stays in supercooled state after expansion, condensation delays even after expansion below saturation line. This is because of the high speed flow and condensation initiates after Wilson zone. In this work the condensation models were tested on CD nozzle geometry. Condensation varies with variation in volumetric ration of volume of steam at exit to inlet[2]. The study of condensation of steam in converging diverging nozzle has been started many years ago, but the focus was on 1D analysis of Laval nozzle. Later 2D calculations were developed to study the real flow behavior in turbine cascades $[3,4,5]$. Equilibrium and non equilibrium numerical models were developed [6].

In this work CFD analysis of flow of wet steam inside CD nozzles is done using Homogeneous Equilibrium Model. The results were compared with the experimental and non equilibrium results of Moore nozzle [4].

\section{NUMERICAL MODELS}

To solve the condensation related problems following thermodynamical models are used.

\subsection{Equilibrium Models}

The most simple mono-pressure model that takes condensation into account is based on Euler equations closed with an equilibrium equation of state:

$$
\begin{gathered}
\frac{\partial \rho_{m}}{\partial t}+\frac{\partial \rho_{m} u_{m, j}}{\partial x_{j}}=0 \\
\frac{\partial \rho_{m} u_{m, i}}{\partial t}+\frac{\partial\left(\rho_{m} u_{m, j} u_{m, i}\right)}{\partial x_{j}}+\frac{\partial p}{\partial x_{i}}=0 \\
\frac{\partial \rho_{m} E_{m}}{\partial t}+\frac{\partial \rho_{m} u_{m, j} H_{m}}{\partial x_{j}}=0
\end{gathered}
$$

Phase change and liquid phase do not occur in these equations but the properties are considered as the thermodynamic mixture of both phases. $p, \rho_{m}, u_{m}, E_{m}, H_{m}$ 
are the pressure, density, velocity, total energy and total enthalpy of mixture respectively. Liquid mass fraction $y$ is used to link the liquid/gaseous phase properties with mixture properties.

Both the liquid and vapor phases are considered to be in thermal equilibrium. When temperature and pressure values drops to saturation value, the condensation appears smoothly and it varies along the liquid vapor equilibrium line.

\subsection{Non Equilibrium Models}

In steam turbines the condensation occurs at non equilibrium condition, flow remains dry even after crossing the saturation line. In this non equilibrium state the flow is said to be in subcooled state until it reaches Wilson point. The position of this point depends on many parameters like pressure of the flow, temperature and expansion rate. When flow reaches Wilson point a condensation shock appears, and a fog of droplets is created. The phase change induces a latent heat release so that the flow comes back to the equilibrium condition.

In this model the liquid and vapor are assumed to be in non equilibrium state. Saturation line is considered to be a band of liquid vapor distribution. The steam stays in subcooled state in this band until it reaches a point after which there will be sudden jump in pressure ratio causing nucleation.

\section{NOZZLE DESCRIPTION}

\subsection{Nozzle Geometry}

Two Moore nozzles are analyzed in this work and the geometries are similar to those used in experimental calculation by Moore [4].

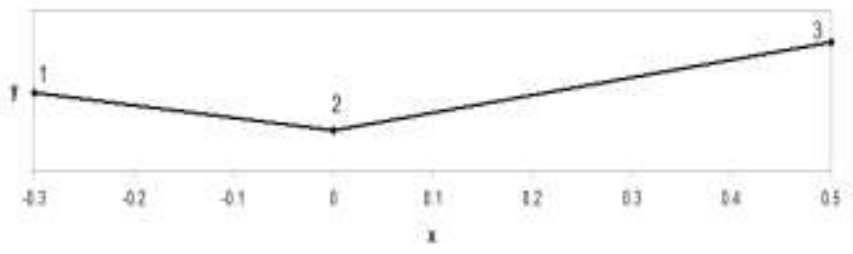

Fig.1 Schematic layout of locations for nozzle geometries

The $x, y$ coordinates of nozzle at respective points are tabulated in following table (All the dimensions are in meters).

Table.1 Cartesian coordinates of major positions in layout of nozzle

\begin{tabular}{|l|l|l|l|l|}
\hline \multicolumn{2}{|l|}{ Position } & 1 & $\mathbf{2}$ & $\mathbf{3}$ \\
\hline \multirow{2}{*}{ Nozzle 1 } & $\mathbf{x}$ & -0.3 & 0.0 & 0.5 \\
\cline { 2 - 5 } & $\mathbf{y}$ & 0.0563 & 0.05 & 0.072 \\
\hline \multirow{2}{*}{ Nozzle 2 } & $\mathbf{x}$ & -0.3 & 0.0 & 0.5 \\
\cline { 2 - 5 } & $\mathbf{y}$ & 0.0663 & 0.06 & 0.075 \\
\hline
\end{tabular}

\subsection{Mesh Details}

Nozzle geometries are generated with the above geometrical coordinates and a quadrilateral mesh is generated. Fine meshing is done near to the wall boundary and near to the throat for better results. The number of grids generated for both nozzles are around 10400 elements with 21400 boundary faces and 20500 internal faces. Schematic views of the grid configuration of both nozzle profiles are displayed in below figures.

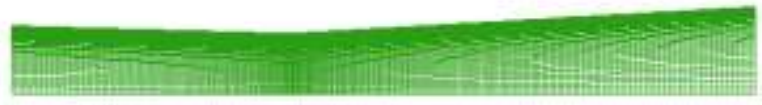

Fig 2. Grid configuration of Nozzle 1

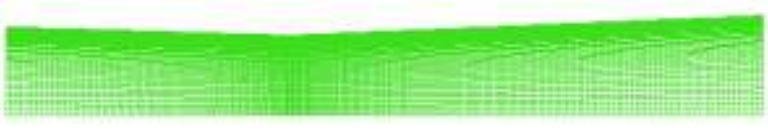

Fig 3. Grid configuration of Nozzle 2

\subsection{Initial and Boundary Conditions}

Initially the flow inside the domain is assumed to be completely dry and considered isentropic expansion of steam. The initial values are taken from steady state values of a normal flow case without condensation.

In boundary conditions flow is assumed to be symmetric along the central axis of the nozzle, hence bottom surface and two parallel longitudinal surfaces are applied with symmetric boundary condition. The entrance converging section is given with inlet, exit of diverging section is given as outlet and the top converging diverging surface is used as adiabatic, no slip wall. To maintain the impermeable wall condition, the mass flux across the wall boundary is set to be zero. For both nozzles the inlet total pressure $p_{o}$ of $25 \mathrm{kPa}$ is used and total temperature $T_{o}$ of $357.6 \mathrm{~K}$ for nozzle 1 and $358.6 \mathrm{~K}$ for nozzle 2 is used. The outlet conditions of the flow must be low enough to maintain supersonic flow at nozzle exit, any high pressure values may cause reverse flow hence these outlet values can be extrapolated from computational domain. The gravity effect and turbulence effects are considered, a turbulence intensity of 0.01 and turbulence dissipation rate of 0.001 is used in inlet boundary condition.

\section{RESULTS AND DISCUSSION}

In this section, primarily a comparative study is conducted with HEM(Homogenous Equilibrium Model) results, Non Equilibrium Model results and available experimental results and later some results of HEM model are discussed for which the experimental values are not available for comparison.

\subsection{Comparison of Variation of Pressure Ratio}

In this work the quantitative validation is carried for HEM model using the experimental values of Moore and non 
equilibrium values of Kermat Fakhari[4]. The comparison between both the numerical models and experimental values pressure ratio distribution along the axis of the nozzle are depicted in below figures.

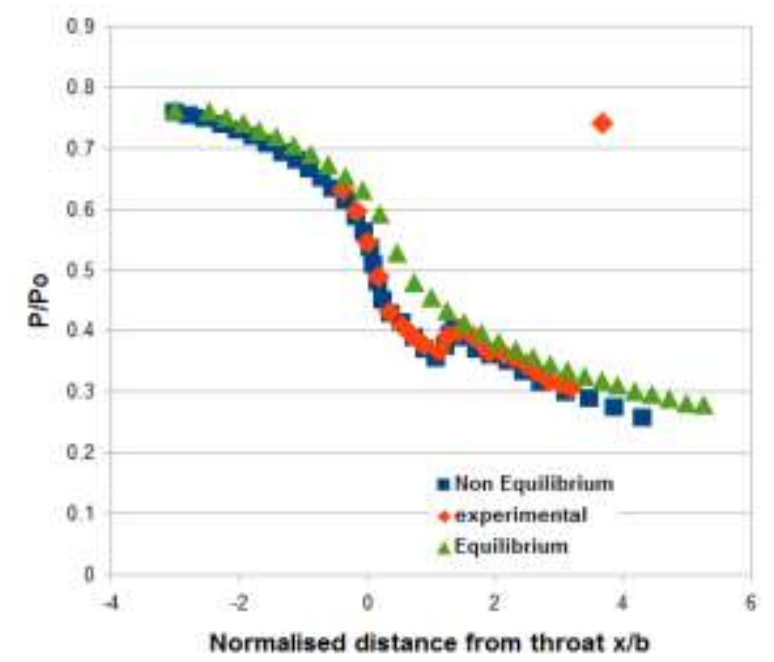

Fig 4. Distribution of pressure ration for Nozzle 1

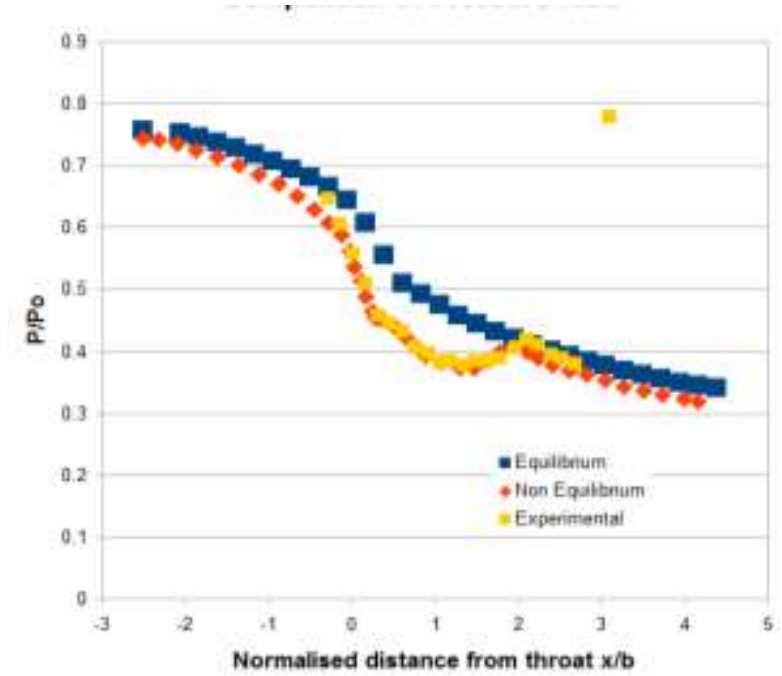

Fig 5. Distribution of pressure ration for Nozzle 2

From Fig 4. we can say that for Nozzle 1 the results of all the three methods are in close agreement in converging section. Near to the throat the HEM curve departs from both experimental and non equilibrium results, this is because of assumption of equilibrium between both phases in HEM. In downstream both theoretical values depart from the measured values. This may be because of boundary layer formation.

Condensation shock appears where the pressure values deviate from isentropic values. This can be seen by sudden jump in the value of pressure ratio. We can see sudden increase in the pressure ratio values in above non equilibrium and experimental curves. In HEM the condensation shock will not be seen because after saturation point, the flow properties vary isentropically on the liquid vapor equilibrium line. From the above figures, we can say that the non equilibrium and experimental results in downstream section for Nozzle 1 are in close agreement compared to Nozzle 2.

\subsection{Comparison of Wetness Fraction}

Wetness fraction is the percent of liquid phase in wet steam. Wetness fraction for steam above saturation pressure and temperature will be zero. Wetness fraction increases when steam expands to a pressure below saturation value. In this work wetness fraction along the length of nozzle using equilibrium model is compared with that of non equilibrium model. The below figures graphically represents comparison between two models,

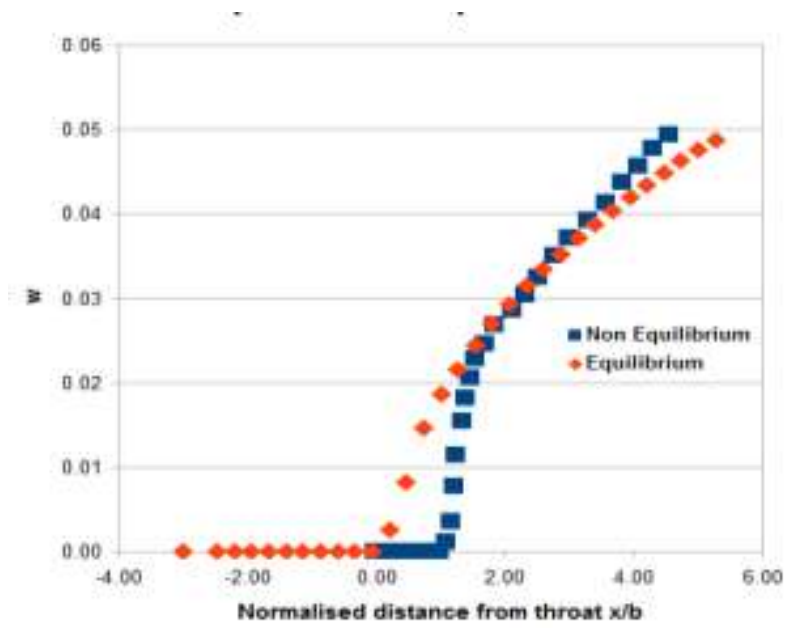

Fig 6. Comparison of wetness fraction for Nozzle 1

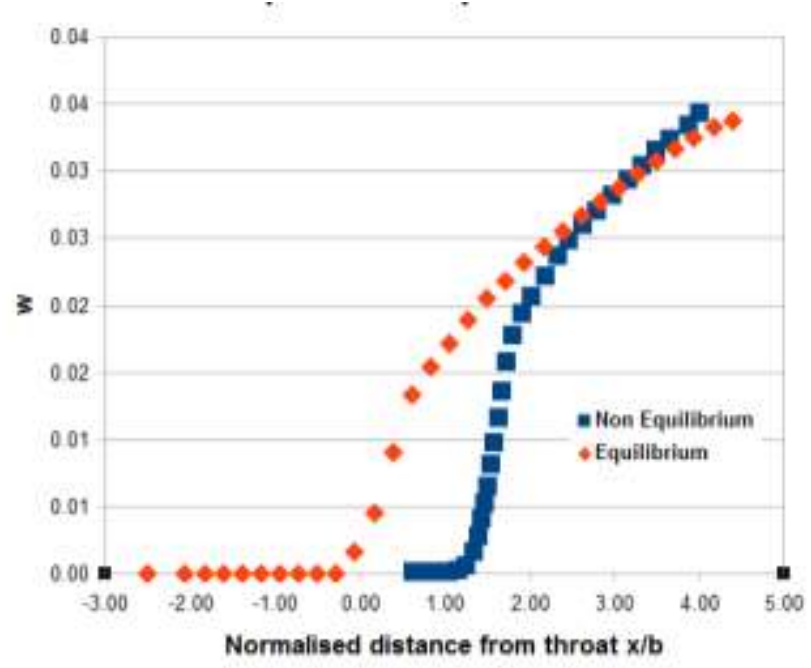

Fig 7. Comparison of wetness fraction for Nozzle 2

The wetness fraction value in case of HEM increases before the throat, where as for non equilibrium model the value remains zero for a little length after the throat. This is because non equilibrium model accounts the properties of vapor in sub cooled state. In the above graphs we can see sudden rise in wetness fraction values for non equilibrium model, which is due to condensation shocks. Condensation shocks appear when steam reaches Wilson point, after which condensation occurs rapidly. There will be no condensation shocks in HEM model because of isentropical variation of 
equilibrium properties hence condensation appears smoothly. The equilibrium line crosses non equilibrium line and shows reduction in wetness, this may be because of rise in local temperature. Following are some of the contour plots of homogenous equilibrium model showing the variations of flow properties.

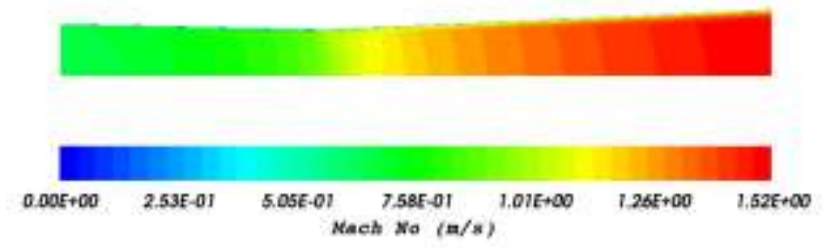

(a) Mach number

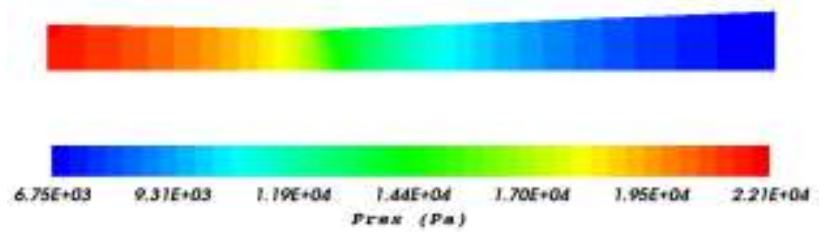

(b) Pressure

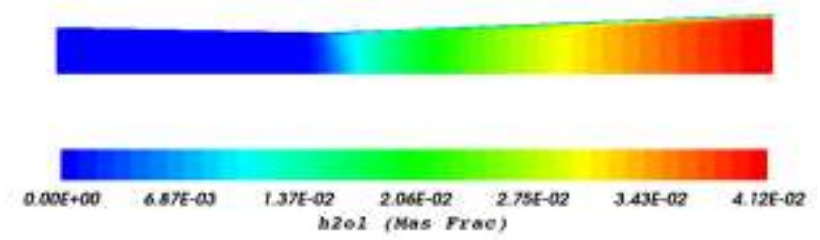

(c) Wetness fraction

Fig 8. For Nozzle 1

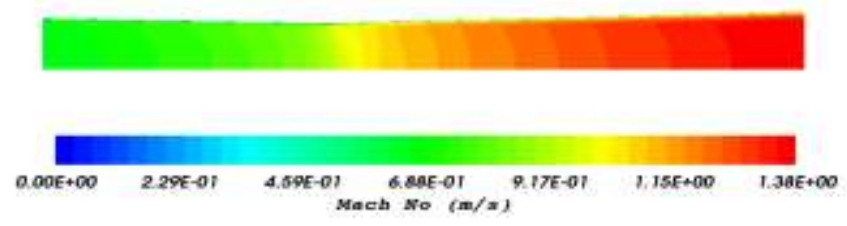

(a) Mach number

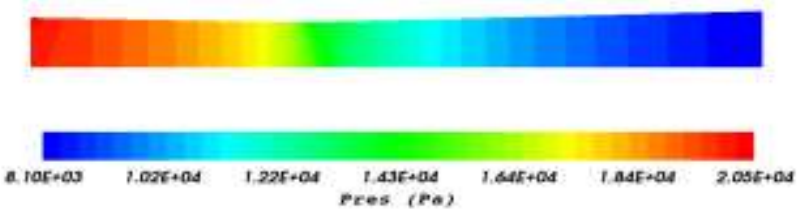

(b) Pressure

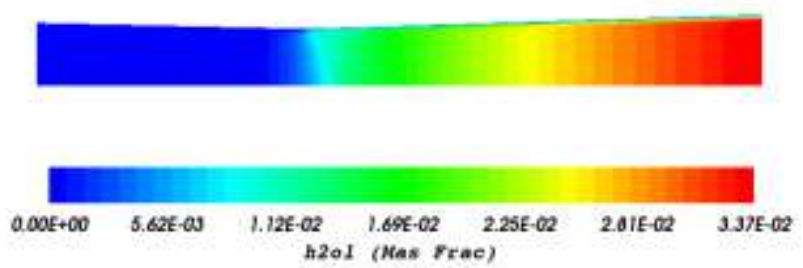

(c) Wetness fraction

\section{CONCLUSION}

A study on numerical models to solve different thermodynamical parameters of condensing flow of steam is carried. Based on the CFD simulation performed for supersonic flow in CD nozzle, the following conclusions can be made.

- The results non equilibrium models were in close agreement with the experimental values compared to HEM values. The pressure ratio variation for nozzle 1 was very close to experimental values compared to nozzle 2.

- The HEM model shows the condensation effects before the throat where as the condensation shock for non equilibrium model appears after the throat, which was same in case of experimental work.

- The non equilibrium models are better compared to HEM models to solve condensing flows in high speed, expansion devices.

\section{ACKNOWLEDGMENT}

This study is supported by Fluidyn Consultancy Pvt Ltd with their software support and valuable guidelines.

\section{REFERENCES}

[1]. Baumann K., (1921), "Some recent developments in large steam tur-bine practice". Journal of Inst. Electr. Eng., Vol. 59, pp. 565-623.

[2]. C.T.R Wilson, "Condensation of water vapor in the presence of dust-free air and other gases", Proc. R. Soc. Lond. 1897 61, 240-242, Published 1 Jan 1897.

[3]. Hasril Hasini, Mohd. Zamri Yusoff and Norhazwani Abd. Malek, "Numerical Modeling of Wet Steam Flow in Steam Turbine Channel", Centre for Advanced Computational Engineering, College of Engineering, University Tenga Nasional, Malaysia.

[4]. Keramat Fakhari, "Numerical Modeling and Investigation of Unsteady Phenomena in Condensing Flows of Industrial Steam Turbines", Fakultät für Maschinenbau der Ruhr-Universität Bochum 2010.

[5]. Danmei Xie, Xinggang Yu, Wangfan Li, Youmin Hou, Yang Shi, Sun Cai, "Numerical Simulation of Water Droplets Deposition on the Last-Stage Stationary Blade of Steam Turbine", Energy and Power Engineering, 2010, 2, 248-253.

[6]. Frederic Blondel, Bruno Audebert, Thomas Pasutto, Mugurel Stanciu, "Condensation models and boundary conditions for non-equilibrium wet steam flows", International Journal of Finite Volumes.

Fig 9. For Nozzle 2 